\title{
Disfemia y ansiedad en el aprendizaje de inglés como lengua extranjera
}

\section{Stuttering and anxiety in English as a foreign language learning}

\section{Resumen}

Este trabajo fundamentalmente cualitativo explora la interrelación entre disfemia, ansiedad en una lengua extranjera (ALE), y aprendizaje de inglés para identificar las áreas de mayor dificultad de aprendizaje en estudiantes disfémicos adolescentes y adultos españoles aprendices de esta lengua. De una muestra de treinta y dos alumnos, dieciséis con disfemia (ACD) y dieciséis sin disfemia (ASD), se analizaron entrevistas realizadas con los primeros, y se compararon sus respuestas con las de Ios ASD a dos escalas de ALE. Los resultados indican que la disfemia y la ALE afectan al aprendizaje lingüístico de los ACD negativamente, provocando el rechazo de su identidad lingüística. La lectura en voz alta y la interacción oral frente al profesor y el grupo clase fueron las áreas más problemáticas con niveles de ALE significativamente mayores en ACD. Ello subraya la necesidad de implementar prácticas pedagógicas que reduzcan la ALE de estos estudiantes y refuercen su auto-concepto.

\section{Palabras clave}

Disfemia, ansiedad en una lengua extranjera (ALE), aprendizaje y enseñanza del inglés como lengua extranjera, destrezas lingüísticas.

\begin{abstract}
This study, which is primarily qualitative, explores the interrelationship between stuttering, foreign language anxiety (FLA), and English as a foreign language learning in order to identify the areas of greatest learning difficulty in stuttering adolescent and adult students who learn this language. From a sample of thirty-two learners, sixteen learners who stutter (LWS) and sixteen learners who do not stutter (LWDNS), interviews that were conducted with the former were analysed, and their responses to two FLA scales were compared to those of the LWDNS. Results indicate that stuttering and FLA negatively affect language learning in LWS, producing rejection of their linguistic identity. Reading aloud and oral interaction in front of the teacher and peers in class were the most problematic areas with significantly higher anxiety levels in LWS. This underscores the need to implement pedagogical practices that reduce these students' FLA, and reinforce their self-concept.
\end{abstract}

\section{Keywords}

Stuttering, foreign language anxiety (FLA), English as a foreign language teaching and learning, language skills.

\author{
María Dolores García Pastor \\ <maria.d.garcia@uv.es> \\ Universidad de Valencia. España
}

\section{Ronan Miller}

<ronanlmiller@gmail.com>

Universidad de Valencia. España

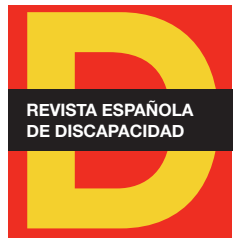

Para citar:

García, M. D. y Miller, R. (2019): "Disfemia y ansiedad en el aprendizaje de inglés como lengua extranjera". Revista Española de Discapacidad, 7 (I): 87-109.

Doi: <https://doi.org/10.5569/23405104.07.01.05>

Fecha de recepción: 12-02-2018 Fecha de aceptación: 25-04-2019 


\section{Introducción}

La disfemia no suele ser considerada socialmente como una discapacidad severa en comparación con otras discapacidades físicas y mentales, y tiende a pasar desapercibida no solamente por la ausencia de evidencia física inmediata de la misma en el individuo disfémico, sino también, en parte, por la gran capacidad de este para ocultarla (Pierre, 2012). Ello explica la relativa escasez de información existente sobre esta discapacidad en comparación con otras a nivel social en general (Álvarez, 2017), y en contextos educativos en particular, especialmente en situaciones de enseñanza y aprendizaje de lenguas extranjeras. En la enseñanza-aprendizaje de inglés como lengua extranjera (ILE) existe pues un desconocimiento sobre cómo la disfemia influye en el aprendizaje lingüístico, por ejemplo, en términos de la ansiedad que los alumnos disfémicos experimentan en el aprendizaje de esta lengua (ansiedad en una lengua extranjera o ALE), y las destrezas lingüísticas más afectadas por este tipo específico de ansiedad, a saber, el habla y la interacción oral, la comprensión auditiva, la lectura y la escritura (Consejo de Europa, 2017).

Sin embargo, muchos estudiantes que aprenden inglés experimentan ansiedad en el aprendizaje de esta lengua extranjera, la cual se potencia en nuestro sistema educativo debido a las exigencias en el dominio de la lengua inglesa que se le imponen al alumnado fundamentalmente en la Educación Superior, en donde ha de acreditar entre un nivel B1 y un nivel B2 de inglés, dependiendo de la titulación y la universidad en la que curse sus estudios (Halbach et al., 2015). Existen además paralelismos claros entre la disfemia y la ALE, por los cuales la primera ha sido descrita como un efecto de la segunda (Horwitz et al., 1986; Price, 1990; Young 1991), y muchos síntomas de ALE (aprensión, preocupación lindante con el temor, sudoración, etc.) se han apreciado de un modo constante y más intenso en los alumnos disfémicos.

A pesar de estos paralelismos, desconocemos por el momento estudios que hayan abordado la relación entre la disfemia y este tipo de ansiedad bien desde la investigación sobre discapacidad y necesidades educativas especiales, bien desde los estudios sobre ALE en la enseñanza y el aprendizaje de lenguas extranjeras. Estos últimos trabajos se han centrado generalmente en investigar la ALE en alumnos convencionales adolescentes y adultos en detrimento de otro tipo de poblaciones estudiantiles como los alumnos disfémicos. Así pues, en este estudio pretendemos explorar, fundamentalmente desde un punto de vista cualitativo, la relación entre la disfemia, la ALE, y el aprendizaje lingüístico en estos alumnos con el objetivo de identificar las áreas de aprendizaje en esta lengua que les producen mayores niveles de ansiedad, y por tanto, mayores dificultades de aprendizaje. De este modo, esperamos contribuir modestamente a la sensibilización del profesorado y del estudiantado en relación con la disfemia en general y en contextos de enseñanza-aprendizaje de ILE en particular, así como a mejorar la comprensión de la ALE en alumnos disfémicos. Esperamos también ofrecer a los docentes de inglés sugerencias para poder atender mejor las necesidades de estos estudiantes, y promover prácticas pedagógicas que disminuyan su ALE y la de todos sus alumnos. 


\section{Disfemia y ansiedad en una lengua extranjera}

La disfemia o tartamudez es un fenómeno que acontece en la comunicación y que desde un punto de vista estrictamente médico constituye un trastorno consistente en un problema individual que solo puede resolverse mediante terapia. Esta concepción de la disfemia focaliza en el individuo y le insta a aceptar su disfemia y concebirla como un problema, enfatizando, la necesidad de tratamiento como solución al mismo (Areheart, 2008). Por tanto, el "modelo médico" que subyace a muchas prácticas institucionales médicas, terapéuticas y educativas en relación con la disfemia la concibe como una discapacidad en tanto en cuanto la define como algo biológico que se basa en las características mentales y físicas que muestran los individuos disfémicos, y por tanto, como algo binario, ya que la presencia o ausencia de dichas características determina si una persona es disfémica o discapacitada o no lo es. Esta visión de la disfemia, y, en general, de la discapacidad, refuerza la percepción del individuo disfémico como alguien que necesita compasión o es un superviviente que ha superado serias dificultades en la vida, y que constituye un modelo a seguir para muchos (ibid.). Aunque algunos individuos disfémicos puedan llegar a aceptar estos roles, es comprensible que muchos otros no se encuentren identificados con los mismos. Así pues, el modelo médico y la visión que promueve de la discapacidad, encasilla a la persona disfémica desde un punto de vista cognitivo, físico y social, y pone "la carga del cambio" en esta y no en el entorno, en donde el planteamiento de determinadas rectificaciones y enseñanzas podría ser necesario (Álvarez, 2017: 11).

Por su parte, un enfoque más social al estudio de la disfemia reconoce a los individuos que la padecen como un grupo social relativamente extenso, si tenemos en cuenta el porcentaje de población disfémica mundial (Yairi y Ambrose, 2013), el cual requiere de la comprensión y de la aceptación de los demás. Este enfoque surge en el Reino Unido en el seno de discusiones sobre política social en torno a la disfemia (Bailey et al., 2015), y como reacción a la visión de la discapacidad como una "tragedia personal" (Oliver, 1986). Este "modelo social" distingue pues entre impedimento, el cual define como una limitación biológica, y por tanto, como algo privado y específico del individuo, y discapacidad, la cual está relacionada con la exclusión social y se concibe como algo público y como parte de los elementos estructurales de la sociedad (Shakespeare, 2006). En este sentido, el modelo social sobre la disfemia permite entender mejor las experiencias de los individuos disfémicos, así como descubrir y comprender las barreras sociales con las que estos se encuentran, las cuales explican ciertos comportamientos y actitudes que se desvían de determinadas normas y expectativas sociales en la persona disfémica. Asimismo, dicho enfoque considera al individuo con disfemia como "sujeto activo de derechos" que reclama a la sociedad su inclusión social dejando al margen concepciones peyorativas y limitantes (Álvarez, 2017). Ello conlleva una visión de la disfemia como otra posibilidad más dentro de un conjunto de comportamientos verbales que tienen lugar en la sociedad, la cual, para algunos autores, constituye una forma de hablar única, legítima y natural igual que otras (Constantino, 2018).

Así pues, en este trabajo hemos adoptado dicho enfoque centrándonos en el tipo más común de disfemia, a saber, la "disfemia evolutiva", la cual tiene su origen en la infancia y no constituye una respuesta a un trauma psicológico u orgánico (Guitar, 2014; Ward, 2017). La disfemia evolutiva aparece entre los dos y cinco años de edad, que se corresponde con el período más intenso que atraviesa el niño en la adquisición del lenguaje. Puede persistir y continuar en la edad adulta o desaparecer de manera parcial o total a medida que este avanza hacia su recuperación con o sin tratamiento. La disfemia evolutiva afecta aproximadamente a un $1 \%$ o un porcentaje menor de los niños en edad escolar y de la población adulta, y prevalece cuatro veces más 
en hombres que en mujeres con un $8 \%$ de incidencia a lo largo de la vida, y una ratio entre ambos decreciente cuanto menor es la edad de los niños (Bloodstein y Ratner, 2008; Yairi y Ambrose, 2013).

A pesar de toda esta información, todavía se desconocen las causas de este trastorno. Existen evidencias de que tiene una base genética, de modo que el individuo disfémico hereda una predisposición neurofisiológica a la disfemia, y que esta tendencia innata se relaciona con las vías neuronales que desarrolla para el habla y el lenguaje en su cerebro. Dicha tendencia combinada con otros factores emocionales (p.ej. una mudanza, el nacimiento de un hermano o hermana en la familia, etc.) puede precipitar la disfemia ${ }^{1}$ en un niño (Guitar, 2014). Las investigaciones sobre disfemia e imagen cerebral han demostrado que las distintas vías neuronales para el habla y el lenguaje que desarrollan los individuos disfémicos proceden de diferencias estructurales y funcionales en sus cerebros (p.ej. Brown et al., 2005; Ingham et al., 2007). Las diferencias estructurales consisten en una sobre-activación de las áreas corticales motoras, una lateralización anómala de las áreas relacionadas con el habla, y la no activación de las áreas auditivas durante la producción del habla; mientras que las diferencias funcionales mayoritariamente hacen referencia a las interrupciones en el flujo del habla que caracterizan a este trastorno.

Estas interrupciones frecuentes e involuntarias, las cuales constituyen repeticiones de fonemas, sílabas, y palabras de una sílaba; prolongaciones de sonidos; y bloqueos en el habla, no son comúnmente aceptadas en una sociedad en la que la fluidez en el habla se espera de cualquier individuo, y se valora en gran medida hasta el punto de asociarse al dominio de una lengua (cf. Ward, 2017). En consecuencia, la disfemia suele producir creencias y emociones negativas en los individuos disfémicos que constituyen los componentes cognitivos y afectivos de este trastorno (Yarus y Quesal, 2006), y les impiden desempeñar muchas tareas cotidianas; construir relaciones sociales significativas y satisfactorias; y rendir tanto en contextos laborales como en contextos educativos, todo lo cual contribuye a deteriorar su calidad de vida (Craig et al., 2009; Crichton-Smith, 2002; Klein y Hood, 2004). Dichas creencias y emociones negativas les llevan a realizar un esfuerzo considerable, y a dedicar gran cantidad de tiempo a anticipar su disfluencia en sus intercambios comunicativos con los demás para evitar ciertos fonemas, palabras, situaciones comunicativas, e incluso personas que provocan o acentúan su tartamudez (Plexico et al., 2009). Como resultado, experimentan una gran fatiga mental que les impide concentrarse y comunicarse de un modo eficaz (Boksem et al., 2005). Tienden además a juzgar erróneamente sus habilidades comunicativas de manera negativa, mientras que valoran las de sus interlocutores de manera positiva (Watson, 1995). Por tanto, los individuos disfémicos tienen más probabilidades de sufrir auto-estigma o estigma social y discriminación; experimentar vergüenza y estrés; y poseer una auto-estima baja, viendo así mermada su salud física en última instancia (Álvarez, 2017; Blood et al., 2003; Boyle y Fearon, 2017).

No es sorprendente pues que muchos individuos disfémicos presenten niveles de ansiedad significativamente más elevados que individuos sin disfemia (Craig y Tran, 2014; Iverach y Rapee, 2014). La ansiedad es la sensación subjetiva de tensión, aprehensión, nerviosismo, y preocupación asociada a la excitación del sistema nervioso autónomo (Szyszka, 2017). Por tanto, constituye un estado emocional y motivacional aversivo que tiene lugar en situaciones amenazantes para el individuo (Eysenck et al., 2007). En contextos de evaluación o de aprendizaje, tradicionalmente se han distinguido la "ansiedad-rasgo" asociada a una propensión innata del individuo a experimentar ansiedad (Spielberger, 1966); la "ansiedad-estado", que constituye una respuesta emocional transitoria del individuo a una situación que experimenta en un momento determinado,

1. En este trabajo utilizamos el término disfemia para referirnos a la disfemia evolutiva. 
por ejemplo, antes de un examen (Spielberger, 1983); y la "ansiedad de situación específica", por la cual un individuo tiene una predisposición a sentir ansiedad en un determinado contexto (Endler y Kocovski, 2001) Esta última constituye, por tanto, una especie de ansiedad rasgo aplicada de manera recurrente a un contexto específico (Maclntyre y Gardner, 1991b). El aprendizaje de una lengua extranjera es un ejemplo de este tipo de contextos, ya que ha dado lugar a una ansiedad específica y única conocida como "ansiedad en una lengua extranjera" o ALE (Horwitz et al., 1986; Maclntyre y Gardner, 1991a, 1991b).

La ALE constituye una de las diferencias individuales o variables del aprendiz que junto a la edad, la aptitud, la personalidad, la motivación, las creencias, y los estilos de aprendizaje influyen en el aprendizaje de una segunda lengua o lengua extranjera (L2/LE). En concreto, es uno de los múltiples estados afectivos que experimentan los aprendices de L2/LE cuando reaccionan a determinadas situaciones de aprendizaje (Ellis, 2001; Scovel, 1978), y ha sido definida como "a distinct complex of self-perceptions, beliefs, feelings, and behaviors related to classroom language learning arising from the uniqueness of the language learning process"2 (Horwitz et al., 1986: 128). La ALE es también un tipo de ansiedad de actuación (performance anxiety), la cual aparece en situaciones de evaluación social como comer, hablar en público, realizar un examen o test, tocar un instrumento musical en público, actuar en un escenario, y aprender una L2/LE (Piechurska-Kuciel, 2008). Así pues, se relaciona con otras ansiedades de actuación, aunque no se compone de las mismas, a saber: la aprehensión por la comunicación, el miedo a la evaluación negativa, y la ansiedad de test (Horwitz, 2017).

La aprehensión por la comunicación hace referencia al miedo a comunicarse con la gente (Horwitz et al., 1986) tanto en situaciones reales como imaginarias. Según Daly (1990), este tipo de ansiedad tiene su origen en una predisposición genética a experimentar ansiedad; la historia personal de un individuo en relación con los refuerzos y castigos relacionados con la comunicación; la frustración del aprendiz ante patrones impredecibles de recompensas y castigos por participar en la misma actividad verbal; y las ineficientes habilidades comunicativas del individuo en edad temprana, así como su exposición a modelos deficientes de comunicación. La aprehensión por la comunicación que surge en los aprendices de L2/LE se debe a la exigencia de comunicar mensajes significativos y conversacionalmente adecuados en un sistema sintáctico, semántico, y fonológico distinto al propio, así como a entender mensajes de otros hablantes utilizando dicho sistema en la interacción social (Horwitz, 1995). La conciencia de su limitada competencia lingüística para atender a todas estas demandas junto con su miedo al fracaso y a los problemas que puedan surgir en la comunicación son las causas de dicha aprehensión, la cual les lleva finalmente a abandonar cualquier intento de comunicarse en la L2/FL (Szyszka, 2017).

El miedo a la evaluación negativa tiene lugar en cualquier tipo de situación en la que el individuo se expone a una evaluación social, por ejemplo, una entrevista de trabajo, y alude a su expectativa de ser evaluado negativamente por los demás, y a su tendencia a evitar este tipo de situaciones. Los individuos que experimentan este tipo de ansiedad tienden a preocuparse en exceso por causar una buena impresión en otros, experimentando con frecuencia mayor ansiedad social que aquellos que no buscan constantemente aprobación (Leary y Kowalski, 1995). En el aprendizaje de L2/LE, el miedo a la evaluación negativa es aquel que siente el alumno a que su pronunciación, palabras, y estructuras en la lengua meta sean juzgadas negativamente por el profesor o profesora, que ha de corregir sus errores, y sus compañeros, que podrían mofarse de sus

2. Traducción de los autores: un compuesto distinto de auto-percepciones, creencias, sentimientos, y conductas relacionadas con el aprendizaje lingüístico en el aula y emergentes de la singularidad del proceso de aprendizaje de una lengua. 
usos lingüísticos incorrectos o inadecuados (Price, 1990; Szyszka, 2017). Los estudiantes adultos son más propicios a experimentar este tipo de ansiedad "porque no pueden presentarse en una nueva lengua de manera tan completa como lo hacen en su lengua nativa” (MacIntyre y Gardner, 1991b: 105).

Finalmente, la ansiedad de test, muy común en nuestra sociedad orientada a los exámenes y los tests (Zeidner y Mathews, 2011), constituye el miedo al fracaso, y la experiencia de ser examinado o evaluado tanto antes como durante o después de la situación evaluativa (Sieber, 1980). En consecuencia, este tipo de ansiedad tiene lugar cuando el individuo cree que sus capacidades intelectuales, motivacionales y sociales son puestas a prueba y superadas por las demandas de un test o situación de evaluación (Zeidner y Mathews, 2011). La ansiedad de test emerge de la interacción entre distintos elementos relacionados con el individuo (p.ej. diferencias individuales en la percepción de amenazas, vulnerabilidad, habilidad para la recuperación, etc.), y la situación evaluativa en cuestión (p.ej. la naturaleza de la tarea a realizar, las limitaciones temporales, el tipo y características del examinador, etc.) (Zeidner, 1998). En contextos de aprendizaje de L2/LE, la ansiedad de test se relaciona, por tanto, con la preocupación y el miedo del estudiante a recibir una mala nota en relación con su competencia lingüística en dicha lengua. De entre los distintos exámenes y tests a los que el alumnado se somete continuamente en estos contextos, los exámenes orales son los que provocan mayores niveles de ansiedad, puesto que combinan la ansiedad de test y la aprehensión por la comunicación (Horwitz et al., 1986). A pesar de que la ansiedad de test es un constructo diferente a la ALE, se ha demostrado empíricamente que este tipo de ansiedad está más fuertemente relacionada con esta que con la aprehensión por la comunicación, y el miedo a la evaluación negativa (Horwitz, 1986; Maclntyre y Gardner, 1991a).

En resumen, La ALE es un tipo de ansiedad específica derivada del intento de atender simultáneamente las demandas lingüísticas, sociales y académicas que supone asimilar nuevos sonidos y reglas en un idioma extranjero, y monitorizar el input y el output en su uso. Dicha ansiedad en niveles elevados produce un procesamiento ineficiente de la información en la realización de una tarea y, en consecuencia, afecta negativamente a la capacidad del estudiante para aprender de forma efectiva (Aida, 1994; Maclntyre y Gardner, 1994; Woodrow, 2006).

\section{Metodología}

\subsection{Participantes}

Los objetivos de este trabajo, a saber, i) explorar la interrelación entre la disfemia, la ALE, y el aprendizaje de ILE desde un enfoque principalmente cualitativo, y ii) medir y comparar los niveles de ALE en ACD y ASD como complemento a lo anterior, justifican el tamaño de la muestra. Esta está constituida por 32 alumnos aprendices de esta lengua, 16 ACD (7 mujeres y 9 varones) y 16 ASD (7 mujeres y 9 varones), de edades comprendidas entre 15 y 40 años, y de nivel socio-económico y educativo similar. Los alumnos disfémicos fueron contactados principalmente a través de la Fundación Española de la Tartamudez tanto para el estudio cualitativo como para el pequeño estudio cuantitativo, mientras que los no-disfémicos fueron contactados solo para este último. Se contó además con una muestra secundaria de 350 ASD que únicamente sirvió para validar la Escala de Ansiedad en Destrezas Lingüísticas Específicas (EADLE) (García-Pastor y Miller, 2019) e interpretar mejor los resultados cuantitativos obtenidos al comparar las puntuaciones sobre ALE de 
los ASD y de los ACD en dicha escala y en la Foreign Language Classroom Anxiety Scale (FLCAS) (Horwitz et al., 1986) (vid. infra). Todos los participantes dieron su consentimiento informado para la recogida y el uso de los datos en este estudio, y se aseguró el anonimato de su identidad y sus respuestas. La Tabla 1 ilustra la distribución de la muestra.

\begin{tabular}{|c|c|c|c|c|c|c|}
\hline & \multicolumn{2}{|c|}{ Género } & \multicolumn{3}{|c|}{ Edad } & \multirow{2}{*}{$\begin{array}{c}\mathbf{N}^{\circ} \text { de participantes } \\
\mathbf{N}=32\end{array}$} \\
\hline & masc. & fem. & mín. & máx. & Media (DT) & \\
\hline ACD & 9 & 7 & 15 & 40 & $29(6,6)$ & 16 \\
\hline ASD & 9 & 7 & 23 & 38 & $26(5,5)$ & 16 \\
\hline
\end{tabular}

Fuente: elaboración propia.

\subsection{Instrumentos y datos}

Los instrumentos utilizados para la recogida de datos en este trabajo fueron, por un lado, 16 entrevistas semi-estructuradas con una duración total de 840 minutos (aproximadamente 45-60 minutos de duración cada una), las cuales se realizaron con el grupo de ACD. Los autores se aseguraron de formular preguntas de diversa índole (narrativas, descriptivas, estructurales, comparativas, y evaluativas) para obtener de los participantes una descripción lo más detallada posible de sus experiencias en relación con su disfemia, su ALE, y su aprendizaje lingüístico en el aula de ILE. Las entrevistas se transcribieron siguiendo parcialmente el sistema de transcripción propuesto por Atkinson y Heritage (1984) en sus investigaciones del análisis conversacional. Los datos obtenidos de estas entrevistas y su análisis forman parte del estudio cualitativo llevado a cabo en este trabajo.

Por otro lado, como apuntábamos anteriormente, se utilizaron dos escalas para medir la ALE tanto en los ACD como en los ASD: la FLCAS y la EADLE o Specific Language Skills Anxiety Scale (SLSAS) (vid. Anexo). Las respuestas de los participantes a ambas escalas sirvieron para complementar la información obtenida en las entrevistas, y junto con su análisis, constituyen el estudio cuantitativo realizado en este trabajo.

A pesar de ciertas críticas relacionadas con el excesivo énfasis en la destreza oral, y la falta de ajuste a determinados contextos de enseñanza-aprendizaje (Woodrow, 2006), la FLCAS constituye el instrumento estándar para medir la ansiedad en el aula de lengua extranjera. Esta escala ha sido traducida a otras lenguas como, por ejemplo, el español (Pérez-Paredes y Martínez-Sánchez, 2001), y su validez y fiabilidad han sido demostradas en numerosas investigaciones (vid. Horwitz, 2017). Esta escala contiene un total de 33 ítems organizados en una escala Likert entre 0 (estoy totalmente de acuerdo) y 5 puntos (estoy totalmente en desacuerdo), en forma de afirmaciones sobre situaciones que pueden inducir ansiedad en el aula de lengua extranjera (p.ej. ítem 3, Tiemblo cuando se que me van a preguntar en clase). La escala permite obtener un nivel global de ALE, así como niveles específicos para los distintos ítems en cada participante.

La EADLE fue diseñada por los autores con el ánimo de complementar la FLCAS, que focaliza principalmente en el habla y la interacción oral. Esta escala es el resultado de un análisis de contenido llevado a cabo sobre 
la FLCAS, y la Overall Assesment of the Speakers Experience of Stuttering Scale (Yaruss y Quesal, 2006), que evalúa las experiencias de hablantes disfémicos, y que también ha sido validada internacionalmente. Se tuvieron en cuenta además las escalas de ansiedad existentes para las distintas destrezas lingüísticas como la comprensión oral (Foreign Language Listening Anxiety Scale o FLLAS) (Kim, 2002), la lectura (Foreign Language Reading Anxiety Scale o FLRAS) (Saito et al., 1999), y la escritura (Second Language Writing Apprehension Test o SLWAS) (Cheng et al., 1999), así como distintos estudios cualitativos sobre disfemia (p.ej. Corcoran y Stuart, 1998; Crichton-Smith, 2002), y las sugerencias de algunos investigadores en relación con el diseño y validación de cuestionarios en el aprendizaje de segundas lenguas (Dörnyei y Taguchi, 2009). La EADLE incluye 35 ítems que se basan en una escala Likert de 5 puntos con 0 indicando "nada", y 5 "muchísimo". Estos ítems se organizan en las diferentes destrezas lingüísticas (habla e interacción oral, comprensión auditiva, lectura y escritura), y aluden a situaciones de aula que pueden provocar ALE (vid. Anexo). Las respuestas a dichos ítems indican una puntuación de ALE global, para cada destreza y para cada ítem en cada individuo, que hemos utilizado para apoyar los resultados del análisis cualitativo de las entrevistas.

\subsection{Análisis}

Para el análisis de las entrevistas se utilizó el programa Atlas.ti (versión 7.5.18), y se siguieron las recomendaciones del Interpretive Phenomenological Analysis (IPA) (Smith et al., 2009). Este tipo de análisis ha sido ampliamente utilizado en investigaciones clínicas para analizar la experiencia subjetiva de diversos pacientes con distintos problemas de salud, y sometidos a distintas condiciones médicas. En el caso de individuos disfémicos, se ha empleado para explorar sus creencias, emociones, actitudes y comportamientos en relación con su disfemia. El objetivo es llegar a comprender la experiencia del individuo disfémico para poder atenderle mejor, reflejando así un enfoque más social hacia la disfemia y su tratamiento.

Así pues, por medio de esta metodología de análisis de datos cualitativo, cada autor llevó a cabo un análisis del contenido de las entrevistas de forma independiente en busca de categorías relacionadas con disfemia, ansiedad, y aprendizaje lingüístico (vid., p.ej. Şimşek y Dörneyi, 2017). Tras varias sesiones conjuntas de comparación y re-definición, dichas categorías fueron establecidas y organizadas en categorías superordinadas y categorías subordinadas como muestra la Figura 1.

El análisis de las respuestas de los ACD y los ASD a las escalas consistió en un análisis cuantitativo descriptivo, en donde se calcularon las medias y las desviaciones típicas de dichas respuestas para todos y cada uno de los ítems, y se realizaron pruebas de $t$ para la comparación de medias en ambos grupos, y el cálculo de la significación en relación con las diferencias halladas. Este análisis sirvió para apoyar el análisis cualitativo de las entrevistas, el cual constituye el foco de este trabajo. 
Figura 1. Categorías superordinadas y subcategorías en las entrevistas

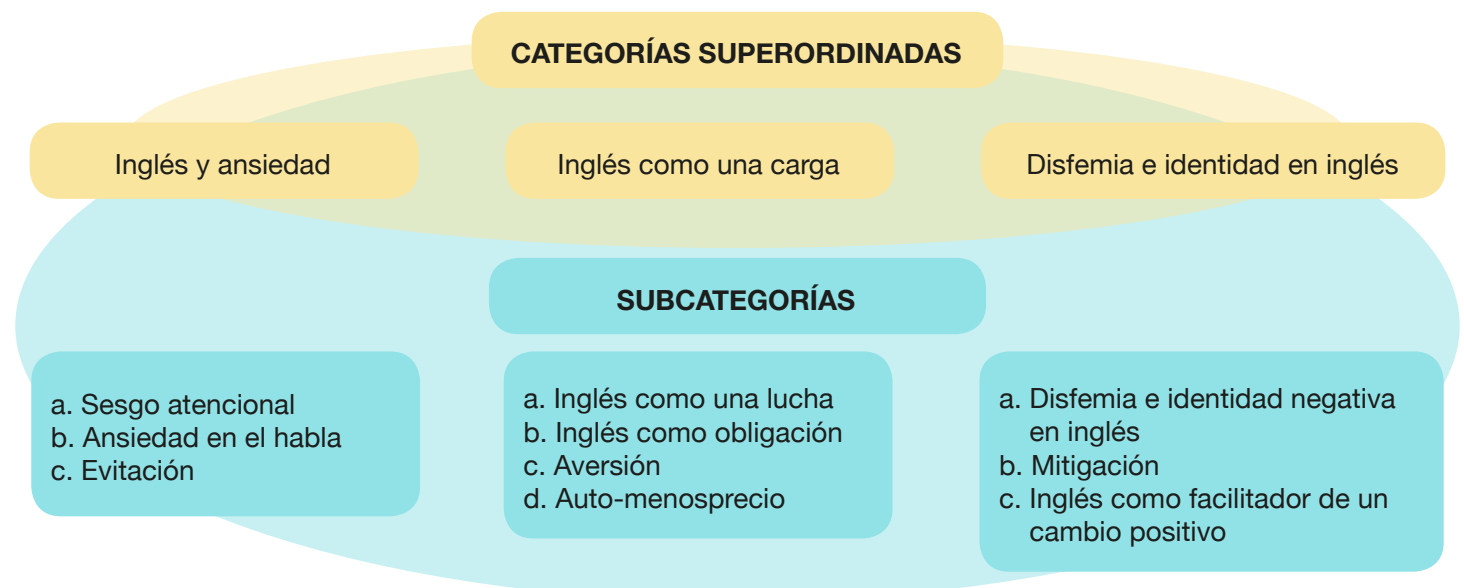

Fuente: elaboración propia.

\section{Resultados}

La relación entre disfemia, ALE y aprendizaje lingüístico en el aprendizaje de ILE que mostró el análisis cualitativo de los datos se ilustra de manera clara en el siguiente ejemplo, que se corresponde con la descripción de dicha interrelación que ofrece EM, varón de 27 años:

"(4.0) Bueno la tartamudez en sí:: no pero la actitud (.) que llevo yo con la tartamudez es decir de NO QUERER HABLAR (.) el estar pendiente siempre de::.: lo que de (.) >bueno de cuando me va a tocar a mi hablar< pues (.) me afecta porque no practico:::: el inglés (.) tanto como debería (.) o no atiendo::: en las clases no atiendo igual que:: >que en cualquier otra clase porque estoy pendiente de:< de lo que voy a decir... Me pone más nervioso (.) entonces el aprendizaje pues claro pues (.) no es igual que (.) que en cualquier otra asignatura” (EM, varón, 27).

En este extracto, la disfemia es la causa de comportamientos y actitudes de EM que impactan negativamente en su aprendizaje lingüístico en un proceso recurrente en el que su deseo de evitar el habla genera aprensión por la expresión oral (Iverach y Rapee 2014; Iverach, et al., 2017), lo cual resulta en una falta de práctica comunicativa que obstaculiza su aprendizaje de la lengua. Ello le produce ALE, que impide su concentración, y dificulta dicho aprendizaje en mayor medida que en otras materias (Szyszka, 2017).

Este ejemplo muestra también las subcategorías "sesgo atencional", que constituye un efecto de la ansiedad, "ansiedad en el habla", que es un tipo de ansiedad, y "evitación" que es un efecto de la ansiedad, las cuales conforman la categoría superordinada "inglés y ansiedad" (Figura 1). Así pues, el sesgo atencional ocurre cuando EM no atiende en las clases de inglés, porque está pendiente de lo que va a decir. Este sesgo apareció de manera recurrente en las experiencias que los ACD describían en relación con sus clases de 
ILE en este trabajo. En individuos disfémicos, el sesgo atencional tiene lugar cuando dirigen su atención a estímulos sociales que perciben como amenazas (Eysenck et al., 2007; Iverach y Rapee 2014; Iverach et al., 2017), juzgando así como intimidatorio el momento de hablar, y dedicando todos sus esfuerzos a anticipar su disfemia (Plexico et al., 2009). En consecuencia, se centran excesivamente en sí mismos, el habla, y los posibles errores que puedan cometer, e infravaloran sus propias intervenciones, todo lo cual les desmotiva en su aprendizaje.

La "ansiedad en el habla" apareció en las entrevistas como la más común frente a otro tipo de ansiedades de actuación como la ansiedad de test (Zeidner, 1998; Zeidner y Mathews, 2011), principalmente en relación con la aprensión por la comunicación, y el miedo a la evaluación negativa. Estos hallazgos no apoyan los resultados empíricos de investigaciones sobre ALE que demuestran la fuerte asociación entre esta y la ansiedad de test en comparación con la aprensión por la comunicación y el miedo a la evaluación negativa (vid. Horwitz, 2017). La ansiedad en el habla también emergió como la dominante frente a otras asociadas a otras destrezas lingüísticas tales como la ansiedad en comprensión auditiva en lengua extranjera (Kim, 2002), la ansiedad en lectura en lengua extranjera (Saito et al., 1999), y la ansiedad en escritura en lengua extranjera (Cheng, et al., 1999). No obstante, los entrevistados identificaron la ansiedad en el habla con la lectura en voz alta en clase (Price, 1990; Rubio-Alcalá, 2017), como muestra el siguiente ejemplo en donde A es la estudiante (23 años) y E es el entrevistador:

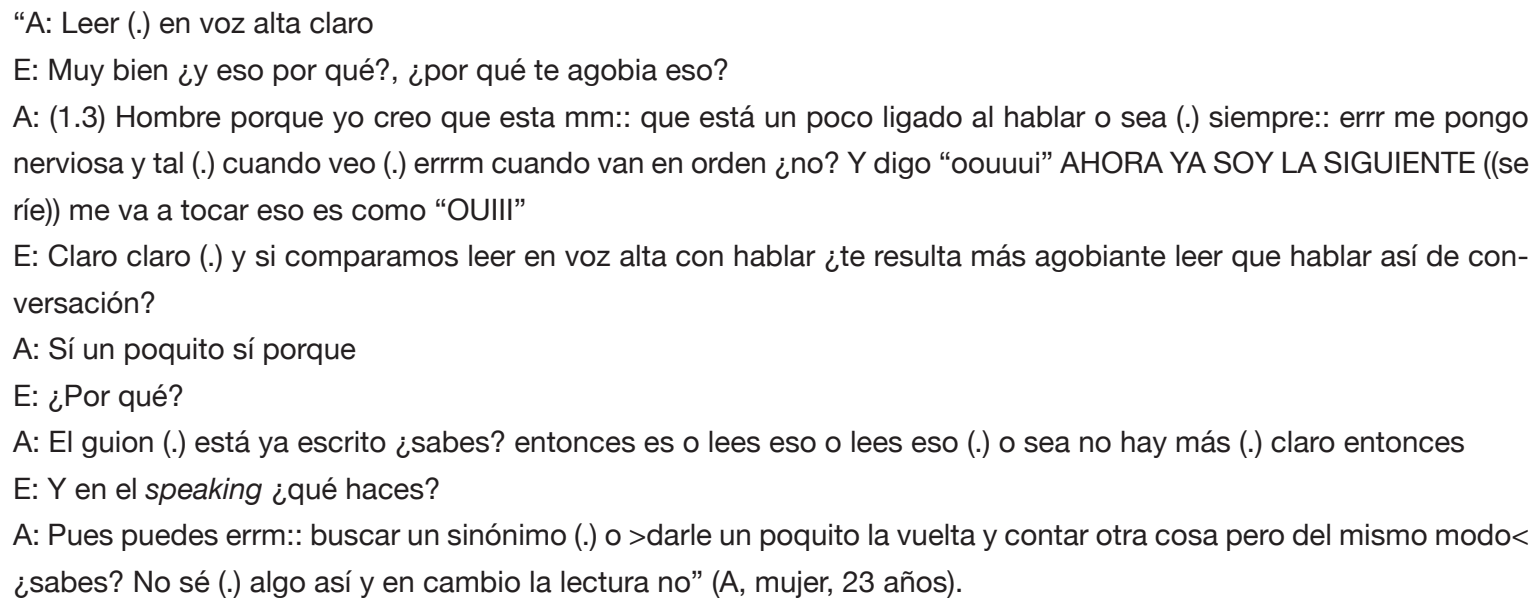

En este ejemplo, $A$ indica un nivel superior de ansiedad en la lectura en voz alta (LVA) que en el habla y la interacción oral (HIO). Aunque relaciona esta modalidad de lectura con la ansiedad en el habla, conceptualmente se refiere a la ansiedad en lectura en lengua extranjera (Saito et al., 1999). Esta surge de la incapacidad de crear la correspondencia símbolo-sonido en un sistema fonético-fonológico alejado de la L1 del hablante, y de entender el significado del texto debido a la falta de conocimiento cultural que subyace al mismo (ibid.). Como refleja este extracto, en ACD dicha incapacidad se acentúa por la imposibilidad de cambiar los elementos del texto por otros que no les ocasionan bloqueos, como suelen hacer normalmente en el habla.

El análisis cuantitativo realizado sobre las respuestas del grupo disfémico y el no-disfémico a las escalas de ALE descritas anteriormente, no mostró, no obstante, que las diferencias para ACD y ASD en la LVA ${ }^{3}$ fueran

3. La FLCAS no contempla la LVA como tal. 
significativas en el estadístico descriptivo de $t$ de Student $(t(30)=1,651, p=0,109)$. Por el contrario, los niveles de ansiedad para aquellos ítems equiparables a la LVA en el habla y la interacción oral (HIO) tanto en la EADLE como en la FLCAS mostraron diferencias significativas para ACD frente a los ASD $(t(30)=3.063, p=0,005$ para la EADLE; y $t(30)=1,344, p=0,189$ para la EAALE) (Tabla 2).

\begin{tabular}{|c|c|c|c|c|c|c|c|c|c|}
\hline & \multicolumn{3}{|c|}{$\begin{array}{l}\text { LVA en EADLE } \\
\text { (Ítems } 3 \text { y } 4 \text { ) }\end{array}$} & \multicolumn{3}{|c|}{$\begin{array}{l}\text { HIO en EADLE } \\
\text { (Ítems } 7 \text { y 9) }\end{array}$} & \multicolumn{3}{|c|}{$\begin{array}{l}\text { HIO en FLCAS } \\
\text { (Ítems } 20 \text { y } 27 \text { ) }\end{array}$} \\
\hline & M & DT & $t$ de Student y $p$ & $\mathbf{M}$ & DT & $t$ de Student y $p$ & M & DT & $t$ de Student y $p$ \\
\hline ACD & 7,9 & 1,6 & \multirow{2}{*}{$\begin{array}{c}1,651 \\
p=0,109\end{array}$} & 5,6 & 1,4 & \multirow{2}{*}{$\begin{array}{c}3,063 \\
p=0,005\end{array}$} & 7,9 & 2,1 & \multirow{2}{*}{$\begin{array}{c}1,344 \\
p=0,189\end{array}$} \\
\hline ASD & 6,8 & 2,2 & & 4,2 & 1,2 & & 7 & 1,5 & \\
\hline
\end{tabular}

Fuente: elaboración propia.

Estos resultados apoyan los hallazgos obtenidos en algunos estudios sobre ALE, en los que aprendices de inglés convencionales sentían menos ansiedad en una clase de lectura en voz alta que en una clase conversacional (Kim, 2009), e indican niveles superiores de ALE en ACD para determinados ítems equiparables a la LVA en la destreza oral. No obstante, en vista de las experiencias relatadas por los participantes disfémicos de este estudio, cabría tener cierta cautela respecto a estos hallazgos, ya que una muestra un poco más amplia de ACD podría llegar a arrojar resultados distintos en el análisis cuantitativo.

Aun así, tanto en la LVA como en el HIO, los entrevistados asocian la ansiedad que experimentan a la corrección de ejercicios gramaticales y a la realización de ejercicios de pronunciación (vid. Rubio-Alcalá, 2017; Szyszka, 2017; Young, 1991). Todo ello sugiere el planteamiento por parte del profesorado de inglés de actividades de lectura e interacción oral alejadas de pedagogías tradicionales, y más acordes con el aprendizaje cooperativo en pequeños grupos, y la co-evaluación y/o evaluación grupal o por parejas. Ello podría contribuir a reducir la ansiedad tanto en los ACD como en los ASD en estas áreas del aprendizaje del inglés.

Aunque la intervención oral frente al profesor y el grupo clase produce niveles de ansiedad elevados en el alumnado en general (Woodrow, 2006), en ACD estos son mayores debido al miedo a ser evaluados negativamente, no solo por un posible deficiente nivel de inglés oral, sino sobre todo por su disfemia, como muestra la siguiente descripción de $\mathrm{N}$ (mujer, 35 años):

"En cuanto al inglés bueno (.) recuerdo que (1.8) siempre: siempre me daba más vergüenza más corte (1.4) hablar en clase (.) imagino que: por:: pues por inseguridad ¿no? por no:: >pues eso< conocer bien el idioma (.) y por la vergüenza de: que me pudiera::: err pt err pues trabar ¿no? por la tartamudez” (N, mujer, 35 años).

Los resultados del estudio cuantitativo para el habla e interacción oral en global apoyan estos hallazgos, de modo que la media de ALE para los ACD fue significativamente mayor en ambas escalas que para los ASD, según los resultados de las dos pruebas de $t$ realizadas $(t(30)=2,072, p=0,047$ en la EADLE; $t(30)=3,176$, $p=0,003$ en la EAALE) (Tabla 3). 


\begin{tabular}{|c|c|c|c|c|c|c|}
\hline \multicolumn{7}{|c|}{ Tabla 3. Totales para HIO en la EADLE y la FLCAS } \\
\hline & \multicolumn{3}{|c|}{$\begin{array}{c}\text { HIO en EADLE } \\
\text { (Ítems 5-16) }\end{array}$} & \multicolumn{3}{|c|}{$\begin{array}{c}\text { HIO en FLCAS } \\
\text { (Ítems } 1,3,9,14,18,20,23,24,27,31,32 \text { ) } \\
\end{array}$} \\
\hline & M & DT & $t$ de Student & M & DT & $t$ de Student \\
\hline ACD & 47,1 & 6,7 & \multirow{2}{*}{$\begin{array}{c}2,072 \\
p=0,047\end{array}$} & 46,6 & 9 & \multirow{2}{*}{$\begin{array}{c}3,176 \\
p=0,003\end{array}$} \\
\hline ASD & 42 & 7,6 & & 36,7 & 8,4 & \\
\hline
\end{tabular}

Fuente: elaboración propia.

En relación con la ansiedad en el habla, los ACD revelaron el desarrollo de determinadas estrategias que les permitían gestionarla y reducirla (Iverach et al., 2017), y que acababan formando parte de su experiencia en el aprendizaje del inglés; por ejemplo: responder lo más rápido posible, mentir indicando desconocimiento de la respuesta, sentarse al final de la clase, esconderse, no participar, no pensar, etc. Muchas de estas estrategias reflejan una tendencia a la evitación tanto mental como física (King y Smith, 2017), que constituye otra subcategoría que identificamos como parte de la categoría superordinada "inglés y ansiedad".

"Inglés como una carga" fue otra categoría superordinada que emergió en las entrevistas, a pesar de que algunos participantes indicaron que no les desagradaba aprender esta lengua. La elevada ALE que sienten los ACD en el aprendizaje del inglés explica su concepción de este como una imposición, concretamente como una lucha y una obligación, que les produce aversión y auto-menosprecio (Rubio-Alcalá, 2017) (vid. Figura 1). Esta concepción de su aprendizaje lingüístico surge de demandas académicas relacionadas con la destreza oral, de la incomprensión del profesorado y parte del alumnado en relación con su disfemia, y de los intensos síntomas fisiológicos de su ALE (p. ej. tics físicos o apretones de dientes), los cuales dificultan una producción oral normal en el idioma extranjero (King y Smith, 2017; Szyszka, 2017). El siguiente extracto de la entrevista con una alumna disfémica $(P$, mujer, 26 años) ilustra estos hallazgos:

«... >el director no< el jefe:.: del inglés del colegio (.) >o jefe del departamento del inglés o como se llame no sé< (.) no sé él pensaba yo que era subnormal ¿no? entonces ((se ríe)) literalmente >en serio< pensaba que yo era un poquito:: (.) cortita no sé por qué pero (.) entonces quien aprobaba el First (.) a ver había como dos niveles de inglés en el colegio (.) en la ESO (.) había como:: > había como< un nivel más bajo y otro más alto ·hh entonces los que aprobaban el First >como que van al otro para prepararse el Advanced< (.) Y YO APROBÉ EL FIRST y nunca me pasó al otro nivel» (P, mujer, 26 años).

La aversión por el aprendizaje del inglés se observó en los ACD a través de la expresión de emociones como el odio o el miedo hacia las clases de esta lengua extranjera, combinadas con una presentación negativa de su identidad como aprendices de lenguas y un menosprecio por la misma (Rubio-Alcalá, 2017), como puede observarse en el siguiente extracto producido por EM (varón, 27 años):

«No sé (.) se me daba muy mal (.) CREO QUE en general los idiomas se me dan muy mal (1.2) ((se aclara la garganta)) y nada me acuerdo (.) bueno en (1.3) primero de bachiller me acuerdo que::: suspendí inglés [...]» (EM, varón, 27).

Esta identidad negativa que ofrecen los ACD aparecía frecuentemente vinculada a su disfemia (Craig et al., 2009), dando lugar a otra de las categorías superordinadas observadas en los datos: "disfemia e identidad 
en inglés". Como apuntan diversos autores, la ALE surge en parte debido al desasosiego que algunos aprendices sienten cuando tienen que interactuar y presentarse a sí mismos de manera auténtica en la lengua extranjera, y son incapaces de hacerlo (Rubio-Alcalá, 2017; Şimşek y Dörnyei, 2017). Se produce así un desencuentro entre "the 'true' self", capaz de expresarse correcta y adecuadamente en L1, y "the more limited self', incapaz de hacer lo propio en L2 (Horwitz et al., 1986: 128). En ACD, este desencuentro no solo tiene lugar en L2, sino también en L1, de modo que su "yo" competente y capaz rara vez logra ver la luz por la imposibilidad que tiene de expresarse de manera fluida en ambas lenguas. En consecuencia, perciben tanto lo que en este trabajo llamamos su "yo en L1" como lo que Dörnyei (2009) acuña con el término "L2 self" (yo en L2) de forma negativa. El siguiente ejemplo producido por C (mujer, 29 años) muestra dicho desencuentro, así como las emociones negativas y la baja auto-estima que llega a provocar:

"Pues muchas veces me sentía mal y triste (.) y sentía que por la tartamudez (.) [...] NUNCA VOY A PODER HABLAR FLUIDO AUNQUE TENGA UN ADVANCED (.) un C1 (.) un C2 (.) nunca voy a poder hablar fluido > pero ya no porque no sepa inglés < (.) sino porque en mi mm en mi idioma materno >tampoco puedo < Y LE DOMINO (.) ... > en las partes que son orales< (.) que son de hablar (.) pues (.) yo no puedo dar >en ese sentido< (.) la talla..." (C, mujer, 29 años).

C expresa así su tristeza y frustración por la imposibilidad de conseguir presentarse de manera auténtica tanto en su L1 como en inglés (Corcoran y Stewart, 1998; Rubio-Alcalá, 2017), ofreciendo una imagen de interlocutora incompetente por su incapacidad de comunicarse oralmente en ambas lenguas de un modo fluido, a pesar de tener los conocimientos para hacerlo. La imposibilidad de interactuar de manera fluida en una lengua, lo cual constituye una demanda social básica y un signo de competencia lingüística en un idioma (Daly, 1990; Ward, 2017), lleva a los alumnos disfémicos a mitigar continuamente la imagen negativa que involuntariamente ofrecen a su interlocutor, así como los posibles efectos negativos de esta y de su disfemia. Para ello, como se observó en los datos, se sirven de estrategias como, por ejemplo, la revelación explícita de su tartamudez a este último, antes de que sea detectada (Collins y Blood, 1990).

A pesar de la negatividad que rodea al aprendizaje del inglés en ACD, muchos de estos estudiantes desean seguir enfrentándose a este como una prueba que han de superar para obtener una visión más positiva de sí mismos. En aquellas ocasiones en las que logran superarla, el aprendizaje de esta lengua extranjera les ofrece la oportunidad de un cambio positivo, alejándoles de su "yo" negativo en L1 y en L2/LE, y aproximándoles a su "ideal L2 self" (yo ideal en L2) (Dörnyei, 2009):

"Ahora aunque salgo nerviosa pero::: salgo:: bien y salgo:: (.) $\mathrm{mmm}$ salgo $\mathrm{mm}$ salgo: pues:: (3.2) salgo (.) pues a veces (.) hasta hasta eufórica (.) sí porque porque estoy haciendo (.) err algo (.) que antes para era: impensable (.) Entonces me siento (.) muy bien [...]" (OC, mujer, 36 años).

\section{Conclusiones}

Como evidencian los resultados expuestos, la relación entre disfemia, ALE, y aprendizaje lingüístico en el aula de ILE en ACD apunta a la disfemia como la causa principal de comportamientos, actitudes, y emociones negativas en estos estudiantes que incrementan su ALE, y, en consecuencia, obstaculizan su apren- 
dizaje lingüístico. Estos incluyen el sesgo atencional; la evitación del habla, en la que indican sentir gran ansiedad; la aversión por esta y el aprendizaje de inglés en general; y el auto-menosprecio, según reveló el análisis cualitativo de las entrevistas realizadas a ACD en este trabajo. La disfemia contribuye pues a aumentar la ALE en estos estudiantes, y esta a su vez acentúa la disfemia en una especie de círculo vicioso que impide un aprendizaje lingüístico efectivo. A pesar de ello, el alumno disfémico no deja de intentar superar sus miedos, y de controlar tanto su disfemia como su ansiedad, así como los efectos perjudiciales que estas le producen a sí mismo y, potencialmente, a su interlocutor, pudiendo llegar a experimentar el aprendizaje de ILE de manera positiva en alguna ocasión. Con ello consigue liberarse temporalmente de su "yo" negativo en L2/LE, alimentando a su vez la esperanza de un cambio similar para su "yo" en L1. Todo ello pone de relieve la dificultad que tienen estos alumnos en su aprendizaje de la lengua extranjera, y, en consecuencia, sus necesidades en el mismo.

Aunque algunos de los comportamientos, actitudes y emociones descritas para los ACD, los cuales se relacionan fundamentalmente con el habla y la interacción oral, podrían observarse en alumnos convencionales que aprenden inglés, el análisis cuantitativo de las respuestas de los ACD y los ASD a la EADLE y la FLCAS para esta destreza mostró niveles de ALE significativamente más elevados en los primeros que en los segundos. De este modo, en condiciones normales, cabría esperar que el nivel de ALE de un alumno disfémico fuese superior al de un ASD.

El habla y la interacción oral con el profesor y frente al grupo clase y la lectura en voz alta aparecieron como las áreas que provocan niveles de ALE significativamente superiores en los ACD que en los ASD, constituyendo, por tanto, las áreas que suponen mayores dificultades de aprendizaje para los primeros. Tanto en la lectura en voz alta como en el habla y la interacción oral, los entrevistados asociaron la ALE a la corrección de ejercicios gramaticales y a la realización de ejercicios de pronunciación, en los que el alumno queda expuesto abiertamente al profesor y los compañeros de clase. Ello sugiere el planteamiento de una pedagogía por parte de los docentes de inglés que incluya actividades de lectura e interacción oral en el aula más colaborativas, de manera que el foco en un determinado individuo quede diluido en el grupo, así como el diseño de tareas que contribuyan a evaluar la competencia oral de los alumnos disfémicos de una manera más justa, y refuercen sus identidades tanto en la lengua extranjera como en su lengua primera. Algunas de estas tareas pueden incluir, por ejemplo, entrevistas orales individuales con el profesor, en las que el alumno conozca de entrada las preguntas que se le van a formular, y disponga de tiempo para preparar su discurso antes y durante la entrevista, o la creación de textos de identidad digitales que muestren la identidad del alumnado y contribuyan a reforzar el vínculo familia-escuela para favorecer un aprendizaje más significativo (p. ej. García-Pastor, 2017, 2018). Solo así se podrá propiciar un ambiente lo suficientemente distendido que facilite el aprendizaje lingüístico no solo a los alumnos disfémicos, sino también al resto del estudiantado.

Finalmente, entre las limitaciones de este estudio se encuentra el tamaño muestral y la falta de realización de un estudio de seguimiento. La ampliación de la muestra y el estudio de seguimiento permitirían conseguir significación estadística, reforzando así el análisis cuantitativo y una mayor visibilidad desde el punto de vista práctico. Asimismo, la extensión de la muestra a otro tipo de estudiantes, como, por ejemplo, alumnos con otras necesidades educativas especiales, o alumnos con disfemia aprendices de otras lenguas extranjeras distintas al inglés, podría constituir una posible vía de investigación futura. La replicación de la investigación con estudiantes de Educación Infantil y Educación Primaria podría además arrojar luz sobre la existencia o 
no de diferencias respecto a las percepciones y experiencias del individuo disfémico en el aprendizaje de la lengua extranjera. En todo caso, la disfemia y la ansiedad constituyen fenómenos que cualquier docente de inglés debería tener en cuenta por su relevancia e impacto negativo en el aprendizaje lingüístico de sus alumnos. En este sentido, el presente trabajo espera haber contribuido modestamente a aportar información útil en esta dirección. 


\section{Referencias bibliográficas}

Aida, Y. (1994): "Examination of Horwitz, Horwitz, and Cope's construct of foreign language anxiety: the case of students of Japanese”. The Modern Language Journal, 78 (2): 155-168.

Álvarez, G. (2017): Las personas con tartamudez en España. Libro Blanco. Madrid: Cermi; Ediciones Cinca.

Areheart, B. A. (2008): "When disability isn't just right: the entrenchment of the medical model of disability and the goldilocks dilemma". Indiana Law Journal, 83 (1): 181-232.

Atkinson, J. M. y Heritage, J. C. (1984): “Transcript notation”, en Atkinson, J. M. y Heritage, J. C. (coords.): Structures of social action: Studies in conversation analysis. Cambridge: Cambridge University Press.

Bailey, K. et al. (2015): "Stammering and the social model of disability: challenge and opportunity". Procedia - Social and Behavioral Sciences, 193: 13-24.

Blood, G. W. et al. (2003): "A preliminary study of self-esteem, stigma, and self-disclosure in adolescents who stutter". Journal of Fluency Disorders, 28 (2): 143-159.

Bloodstein, O. y Ratner, N. (2008): A handbook on stuttering. New York: Thomson Delmar Learning.

Boksem, M. A. et al. (2005): "Effects of mental fatigue on attention: an ERP study". Cognitive Brain Research, 25 (1): 107-116.

Boyle, M. P. y Fearon, A. N. (2017): "Self-stigma and its associations with stress, physical health, and health care satisfaction in adults who stutter." Journal of Fluency Disorders, 56: 112-121 (en línea). <https://www.sciencedirect.com/science/article/pii/S0094730X17300487?via\%3Dihub>, acceso 26 de diciembre de 2017.

Brown, S, et al. (2005): "Stuttered and fluent speech productions: an ALE metanalysis of functional neuroimaging studies”. Human Brain Mapping, 25 (1): 105-117.

Cheng, Y. S. et al. (1999): "Language anxiety: differentiating writing and speaking components". Language Learning, 49 (3): 417-446.

Collins, C. R. y Blood, G. W. (1990): "Acknowledgment and severity of stuttering as factors influencing nonstutterers' perceptions of stutterers”. Journal of Speech and Hearing Disorders, 55 (1): 75-81.

Consejo de Europa (2017): Common European framework of reference for languages: learning, teaching, assessment. Companion volume with new descriptors (en línea). <https:/www.coe.int/en/web/portfolio/thecommon-european-framework-of-reference-for-languages-learning-teaching-assessment-cefr->, acceso 11 de noviembre de 2017.

Constantino, C. D. (2018): "What can stutterers learn from the neurodiversity movement?". Seminars in Speech and Language, 39 (4): 382-396.

Corcoran, J. A. y Stewart, M. (1998): "Stories of stuttering: a qualitative analysis of interview narratives". Journal of Fluency Disorders, 23 (4): 247-264.

Craig, A. et al. (2009): "The impact of stuttering on the quality of life in adults who stutter". Journal of Fluency Disorders, 34 (2): 61-71.

Craig, A. y Tran, Y. (2014): "Trait and social anxiety in adults with chronic stuttering: conclusions following metaanalysis". Journal of Fluency Disorders, 40 (1): 35-43. 
Crichton-Smith, I. (2002): "Communicating in the real world: accounts from people who stammer". Journal of Fluency Disorders, 27 (4): 333-352.

Daly, J. (1990): "Understanding communication apprehension: an introduction for language educators", en Horwitz, E. K., y Young, D. J. (coords.): Language anxiety: From theory and research to classroom implications. Englewood Cliffs: Prentice Hall.

Dörnyei, Z. (2009): “The L2 motivational self system”, en Dörnyei, Z. y Ushioda, E. (eds.): Motivation, language identity and the $L 2$ self. Bristol: Multilingual Matters.

Dörnyei, Z. y Taguchi, T. (2009): Questionnaires in second language research: Construction, administration, and processing. New York: Routledge.

Ellis, R. (2001): The study of second language acquisition. Oxford: Oxford University Press.

Endler, N. S. y Kocovski, N. L. (2001): "State and trait anxiety revisited”. Journal of Anxiety Disorders, 15 (3): 231 245.

Eysenck, M. W. et al. (2007): “Anxiety and cognitive performance: attentional control theory”. Emotion, 7 (2): $336-$ 353.

García-Pastor, M. D. (2018): "Textos de identidad digitales: una valiosa herramienta para el estudio de la construcción de la identidad y el aprendizaje lingüístico en ILE”. Signos, 51: 24-44.

García-Pastor, M. D. (2017): “Learners' identities at stake: digital identity texts in the EFL classroom”. Language Value, 9 (1): 36-61.

García-Pastor, M. D. y Miller, R. (2019): "Unveiling the needs of students who stutter in the language skills - a study on anxiety and stuttering in EFL learning". European Journal of Special Needs Education, 34 (2): 172-188.

Guitar, B. (2014): Stuttering: An integrated approach to its nature and treatment. Philadelphia: Lippincott Williams \& Wilkins.

Halbach, A. et al. (2015): La acreditación del nivel de lengua inglesa en las universidades españolas: actualización 2015 (en línea). <https://www.researchgate.net/publication/275272956_LA_ACREDITACION_DEL_NIVEL_DE_LENGUA_INGLESA_EN_LAS_UNIVERSIDADES_ESPANOLAS_ACTUALIZACION_2015>, acceso 13 julio de 2017.

Horwitz, E. K. (2017): "On the misreading of Horwitz, Horwitz and Cope (1986) and the need to balance anxiety research and the experiences of anxious language learners", en Gkonou, C. et al. (eds.): New insights into language anxiety: Theory, research and educational implications. Bristol: Blue Ridge Summit.

Horwitz, E. K. (1995): "Student affective reactions and the teaching and learning of foreign languages". International Journal of Educational Research, 23 (7): 573-579.

Horwitz, E. K. (1986): "Preliminary evidence for the reliability and validity of a foreign language anxiety scale". TESOL Quarterly, 20 (3): 559-562.

Horwitz, E. K. et al. (1986): “Foreign language classroom anxiety”. The Modern Language Journal, 70 (2): 125-132.

Ingham, R. J. et al. (2007): "Neuroimaging: contributions to developmental stuttering theory and treatment", en Ingham, R. J. (eds.): Neuroimaging in communication sciences and disorders. New York: Plural Publishing.

Iverach, L. y Rapee, R. M. (2014): "Social anxiety disorder and stuttering: current status and future directions". Journal of Fluency Disorders, 40 (1): 69-82. 
Iverach, L. et al. (2017): "Maintenance of social anxiety in stuttering: a cognitive-behavioral model". American Journal of Speech-Language Pathology, 26 (2): 540-556.

Kim, J. (2002): “Anxiety and foreign language listening”. English Teaching, 57 (2): 3-34.

Kim, S-Y. (2009): "Questioning the stability of foreign language classroom anxiety and motivation across different classroom contexts”. Foreign Language Annals, 42 (1): 138-157.

King, J. y Smith, L. (2017): "Social anxiety and silence in Japan's tertiary foreign language classrooms", en Gkonou, C. et al. (eds.): New insights into language anxiety: Theory, research and educational implications. Bristol: Multilingual Matters.

Klein, J. F. y Hood, S. B. (2004): "The impact of stuttering on employment opportunities and job performance". Journal of Fluency Disorders, 29 (4): 255-273.

Leary, M. R. y Kowalski. R. M. (1995): "The self-presentation model of social phobia”, en Heimburg, R. G. (eds.): Social phobia: Diagnosis, assessment, and treatment. London: The Guilford Press.

Maclntyre, P. D. y Gardner, R. C. (1994): "The subtle effects of language anxiety on cognitive processing in the second language". Language Learning, 44 (2): 283-305.

Maclntyre, P. D. y Gardner, R. C. (1991a): "Language anxiety: its relationship to other anxieties and to processing in native and second languages". Language Learning, 41 (4): 513-534.

Maclntyre, P. D. y Gardner, R. C. (1991b): "Methods and results in the study of anxiety and language learning: a review of the literature". Language learning, 41 (1): 85-117.

Oliver, M. (1986): "Social policy and disability: some theoretical issues". Disability, Handicap \& Society, 1 (1): 5-17.

Pérez-Paredes, P. y Martínez-Sánchez, F. (2001): "A Spanish version of the foreign language classroom anxiety scale: revisiting Aida's factor analysis”. Revista Española de Lingüística Aplicada (RESLA), 14: 337-352.

Piechurska-Kuciel, E. (2008): Language anxiety in secondary grammar school students. Opole: Uniwersytet Opolski.

Pierre, J. St. (2012): "The construction of the disabled speaker: locating stuttering in disability studies". Canadian Journal of Disability Studies, 1 (3): 1-21.

Plexico, L.W. et al. (2009): "Coping responses by adults who stutter: part I. Protecting the self and others". Journal of Fluency Disorders, 34 (2): 87-107.

Price, M. L. (1990): "The subjective experience of foreign language anxiety: interview with highly anxious students", en Horwitz, E. K. y Young, D. J. (eds.): Language anxiety: From theory and research to classroom implications. Englewood Cliffs: Prentice Hall.

Rubio-Alcalá, F. D. (2017): "The links between self-esteem and language anxiety and implications for the classroom", en Gkonou, C. et al. (eds.): New insights into language anxiety: theory, research and educational implications. Bristol: Multilingual Matters.

Saito, Y. et al. (1999): "Foreign language reading anxiety". The Modern Language Journal, 83 (2): 202-218.

Shakespeare, T. (2006): “The social model of disability”, en Davis, J. L. (ed.): The disability studies reader. London: Routledge.

Scovel, T. (1978): "The effect of affect on foreign language learning: a review of the anxiety research". Language Learning, 28 (1): 129-142. 
Sieber, J. E. (1980): "Defining test anxiety: problems and approaches", en Sarason, I. G. (ed.): Test anxiety: Theory, research, and applications. Hillsdale: Lawrence Erlbaum Associates.

Şimşek, E. y Dörnyei, Z. (2017): “Anxiety and L2 self-images: the 'anxious self'”, en Gkonou, C. et al. (eds.): New insights into language anxiety: Theory, research and educational implications. Bristol: Multilingual Matters.

Smith, J. A. et al. (2009): Interpretative phenomenological analysis: Theory, method and research. London: Sage.

Spielberger, C. (1966): "Theory and research of anxiety", en Spielberger, C (ed.): Anxiety and behaviour. New York: Academic Press.

Spielberger, C. D. (1983): Manual for the State-Trait Anxiety Inventory (Form Y). Palo Alto: Consulting Psychologists Press.

Szyszka, M. (2017): Pronunciation learning strategies and language anxiety. Amsterdam: Springer.

Ward, D. (2017): Stuttering and cluttering: Frameworks for understanding and treatment. New York: Psychology Press.

Watson, J. B. (1995): “Exploring the attitudes of adults who stutter". Journal of Communication Disorders, 28 (2): 143-164.

Woodrow, L. (2006): “Anxiety and speaking English as a second language”. RELC Journal, 37 (3): 308-328.

Yairi, E. y Ambrose, N. (2013): “Epidemiology of stuttering: 21st century advances”. Journal of Fluency Disorders, 38 (2): 66-87.

Yaruss, J. S. y Quesal, R. W. (2006): “Overall Assessment of the Speaker's Experience of Stuttering (OASES): documenting multiple outcomes in stuttering treatment". Journal of Fluency Disorders, 31 (2): 90-115.

Young, D. J. (1991): "Creating a low-anxiety classroom environment: what does language anxiety research suggest?”. The Modern Language Journal, 75 (4): 426-437.

Zeidner, M. (1998): Test anxiety: The state of the art. New York: Plenum Press.

Zeidner, M. y Mathews, G. (2011): Anxiety 101. New York: Springer. 
Anexo

\section{- Escala de Ansiedad en Destrezas Lingüísticas Específicas (EADLE)}

Edad: Genero:

Las siguientes afirmaciones se refieren a diversas situaciones frecuentes en el aprendizaje de un idioma. Tu tarea consiste en valorar el nivel de ansiedad que te provoca cada situación, utilizando para ello la siguiente escala:

\begin{tabular}{|c|c|c|c|c|}
\hline Nada & No mucho & Más o menos & Mucho & Muchísimo \\
\hline 1 & 2 & 3 & 4 & 5 \\
\hline
\end{tabular}

\section{Cuando lees (reading) en inglés, ¿qué actividades te ponen más nervioso?}

1. Leer para ti y luego contestar preguntas de comprensión lectora en clase para entregárselas luego al profesor.

2. Leer para ti y contestar preguntas de comprensión lectora en clase como parte de un examen.

3. Leer en voz alta y contestar preguntas de comprensión lectora en clase para que el profesor las corrija delante de todos.

4. Leer en voz alta delante de los compañeros de clase para que el profesor evalúe tu pronunciación.

\section{Cuanto hablas (speaking) en inglés, ¿qué actividades te ponen más nervioso?}

5. Exponer un tema en clase elegido por ti que hayas preparado anteriormente (con notas, fichas, powerpoint, etc).

6. Exponer un tema en clase en grupo que hayáis preparado anteriormente (con apuntes, fichas, powerpoint, etc).

7. Hacer ejercicios de repetición de palabras o frases con el resto de la clase para practicar la pronunciación siguiendo al profesor.

8. Cantar en inglés con el resto de la clase para aprender vocabulario, gramática, etc.

9. Responder a preguntas en clase que te hace el profesor.

10. Hablar en grupos pequeños en clase mientras el profesor supervisa los grupos.

11. Escenificar una conversación (role play) en clase con otros alumnos.

12. Hablar con un hablante nativo de inglés por skype u otra plataforma de videochat.

13. Hablar por teléfono en inglés con un hablante nativo. $\square$ 
14. Hacer un examen oral con tu profesor sobre un tema que hayas preparado anteriormente.

15. Hacer un examen oral con tu profesor sobre un tema que no hayas preparado anteriormente.

16. Participar en una discusión en grupo que el profesor evalúa en clase.

\section{Cuando escuchas (listening) en inglés ¿qué actividades te ponen más nervioso?}

17. Escuchar un CD para luego contestar preguntas escritas en clase.

18. 18. Escuchar un $C D$ para contestar preguntas escritas en clase que sean parte de un examen.

19. 19. Escuchar al profesor cuando explica cómo hacer ciertas actividades, tareas, los deberes, etc., en clase.

20. Escuchar a otros compañeros de clase hablando en inglés.

21. Escuchar a un hablante nativo invitado en clase.

22. Escuchar a un hablante nativo en un vídeo o en Internet en clase (ej. Ted Talks, Youtube, etc.).

Cuando escribes (writing) en inglés ¿Qué actividades te ponen más nervioso?

23. Escribir una carta a un amigo en clase que será evaluada por el profesor.

24. Escribir una queja en clase que será evaluada por el profesor.

25. Escribir una solicitud para un puesto de trabajo en clase que luego será evaluada por el profesor.

26. Escribir un texto sobre un tema elegido por ti en clase que luego será evaluado por el profesor.

27. Escribir un texto en clase como parte de un examen.

28. Escribir un correo electrónico en inglés a tu profesor para preguntarle algo.

29. Escribir un correo electrónico a un hablante nativo.

30. Participar en un chat online en inglés con hablantes nativos.

\section{En cuanto a tu opinión sobre el inglés:}

31. ¿Crees que haces el mismo esfuerzo en las clases de inglés que en otras asignaturas?

32. ¿Te imaginas usando inglés a menudo en el futuro?

33. ¿Te imaginas usando inglés en un trabajo en el futuro?

34. ¿Te imaginas hablando inglés en tu vida personal o profesional en el futuro?

35. ¿Crees que el inglés es importante para que logres cosas que consideras importantes en la vida? 


\section{- Foreign Language Classroom Anxiety Scale}

Edad: Genero:

Las siguientes afirmaciones se refieren a diversas situaciones frecuentes en el aprendizaje de un idioma. Tu tarea consiste en valorar tu grado de acuerdo o desacuerdo con cada una de las siguientes afirmaciones, utilizando para ello la siguiente escala:

\begin{tabular}{|c|l|}
\hline 1 & Estoy totalmente de acuerdo \\
\hline 2 & Estoy de acuerdo \\
\hline 3 & No sé \\
\hline 4 & No estoy de acuerdo \\
\hline 5 & Estoy totalmente en desacuerdo \\
\hline
\end{tabular}

1. Nunca estoy completamente seguro de mí mismo cuando hablo en la clase de inglés.

2. No me preocupa cometer errores en clase.

3. Tiemblo cuando sé que me van a preguntar en clase.

4. Me asusta no entender lo que el profesor está diciendo en inglés.

5. No me molestaría en absoluto asistir a más clases de inglés. $\square$

6. Durante la clase, me doy cuenta que pienso en cosas que no tienen nada que ver con la clase.

7. Pienso que a los otros compañeros se les da mejor el inglés que a mí.

8. Normalmente estoy a gusto cuando hago exámenes en clase.

9. Me pongo muy nervioso cuando tengo que hablar en clase y no me he preparado bien.

10. Me preocupa las consecuencias que pueda traer el suspender.

11. No entiendo por qué alguna gente se siente tan mal por las clases de inglés.

12. En clase, me pongo tan nervioso que se me olvidan algunas cosas que sé.

13. Me da corte salir voluntario en clase.

14. Creo que no me pondría nervioso si hablara en inglés con una persona nativa.

15. Me irrita no entender lo que el profesor está corrigiendo.

16. Aunque vaya con la clase preparada, me siento nervioso.

17. A menudo no me apetece ir a clase.

18. Me siento seguro a la hora de hablar en la clase.

19. Me da miedo que mi profesor corrija cada fallo que cometo.

20. Siento como mi corazón palpita cuando sé que me van a pedir que intervenga en clase.

21. Cuanto más estudio, más me lío. 
22. No tengo ninguna presión ni preocupaciones para prepararme bien las clases.

23. Tengo la sensación de que mis compañeros hablan el inglés mejor que yo.

24. Me da mucho corte hablar en la lengua extranjera delante de mis compañeros.

25. Las clases transcurren con tal rapidez que me preocupa quedarme atrasado.

26. Comparativamente, estoy más tenso y me siento más nervioso en la clase de inglés que en otras clases o que en mi propio trabajo.

27. Me pongo nervioso mientras hablo en clase.

28. Antes de entrar a clase, me siento seguro y relajado.

29. Me pongo nervioso cuando no entiendo cada una de las palabras que mi profesor dice.

30. Me abruma la cantidad de cosas que hay que aprender para poder hablar inglés.

31. Temo que mis compañeros de clase se ríen de mí cuando hablo en inglés.

32. Creo que me sentiría a gusto hablando entre nativos que hablan inglés.

33. Me pongo nervioso cuando el profesor pregunta cosas que no me he podido preparar.

\section{- Símbolos de transcripción}

(.) pausa inferior a un segundo

(1.2) pausa con indicación de tiempo en segundos

<> disminución del ritmo del habla

$>\quad$ aumento del ritmo del habla

::: expansión de la consonante o vocal precedente AUMENTO DEL VOLUMEN

thh inhalación

hhh exhalación

(( )) información contextual 